\title{
Case Report \\ Rubinstein-Taybi Syndrome: A Case Report
}

\author{
A. P. Münevveroglu and B. B. Akgöl \\ Department of Pedodontics, Faculty of Dentistry, Istanbul Medipol University, Fatih, 34093 Istanbul, Turkey
}

Correspondence should be addressed to A. P. Münevveroglu, apatir@medipol.edu.tr

Received 12 July 2012; Accepted 8 August 2012

Academic Editors: A. Epivatianos and A. L. S. Guimaraes

Copyright ( 2012 A. P. Münevveroglu and B. B. Akgöl. This is an open access article distributed under the Creative Commons Attribution License, which permits unrestricted use, distribution, and reproduction in any medium, provided the original work is properly cited.

\begin{abstract}
Rubinstein-Taybi syndrome or Broad Thumb-Hallux syndrome is a genetic disorder characterized by facial dysmorphism, growth retardation, and mental deficiency. A seven-year-old girl had come to the Department of Pedodontics, Istanbul Medipol University, Faculty of Dentistry, Turkey, with a complaint of caries and bleeding of gingivae. The patient was mentally retarded. Extraoral features revealed distinctive facial appearance with a broad fore head, hypertelorism, broad nasal bridge, and beaked nose. Intraoral features observed were talons cusps in the upper lateral incisors, carious teeth, and plaque accumulation. Since the patient was mentally retarded, the dental treatment was done under GA. The treatment plan and dental management of this patient are discussed in this case report.
\end{abstract}

\section{Introduction}

Rubinstein-Taybi Syndrome (RTS:OMIM 180849), or Broad Thumb-Hallux syndrome, was initially described by Michail et al. in 1957 [1]. In 1963, Rubenstein and Taybi reported on seven cases of this syndrome [2], which included a group of congenital anomalies consisting of short, broad thumbs and great toes, psychomotor retardation, highly arched palates, and histories of recurrent respiratory infections and particular facial abnormalities [3-5]. It is caused by either a microdeletion at $16 \mathrm{p} 13.3$ or mutations in the CREB-binding protein (CREBBP or CBP) or EP300 gene (at 22q13) [4-7].

The incidence of the syndrome has been estimated to be 1 in every 300,000 newborns [7]. There is an equal male and female incidence [8-10].

The main distinctive features most commonly linked to this syndrome are downward sloping palpebral fissures, broad thumbs and halluces, growth retardation and psychomotor developmental delay, typical facial dysmorphism, hypertelorism, a prominent forehead, and mental disability [9-11].

One intriguing phenomenon is the fact that RTS patients are prone to develop tumours. These tumours show a pattern of neural and developmental origin, and neuroblastoma, medullablastoma, oligodendroglioma, meningioma, seminoma, odontoma, choristoma, and polimatrixtomas were the reported tumours in RTS patients $[3,12,13]$.

Congenital anomalies of cardiovascular system are also described such as ventricular septal defect, patent ductus arteriosus, coarctation and stenosis of the aorta, and pulmonic stenosis $[3,14,15]$.

Oral manifestations of this syndrome include limited mouth opening, a pouting lower lip, retro/micrognathia, a high arched and narrow palate, cleft uvula and palate, and rarely a cleft upper lip. Dental abnormalities occur in $67 \%$ of individuals with RTS and can include hypodontia, maintenance of deciduous teeth, talon cusps, and enamel hypoplasia. An increased rate of caries and periodontal disease has been reported in these patients. However periodontal disease has not always been associated with this syndrome $[7,11,16]$. Characteristics of RTS are described in Table 1.

Dental treatment is generally complicated due to difficulties in managing the patient's behavior. In most patients with this syndrome, it is necessary to carry out the dental treatment under sedation or general anesthesia. It is important to know that these patients might have upper respiratory obstruction during sleeping or sedation because of the anatomical characteristics of their maxillofacial region [1618]. 
TABLE 1: Characteristics of RTS.

\begin{tabular}{ll}
\hline Orthopedic & Broad thumbs, first toes, short stature, vertebral abnormalities \\
Eye & Strabismus, refractory errors, ptosis, coloboma, ptosis, cataracts, nystagmus \\
Cardiac & Congenital heart defects \\
Dental & Crowding teeth, malocclusion, multiple caries, hypodontia, hyperdontia, talon cusps \\
Tumors & Meningioma, neuroblastoma, medullablastoma, oligodendroglioma, seminoma \\
Skin & Keloids \\
Sleep apnea & Obstructive sleep apnea \\
\hline
\end{tabular}

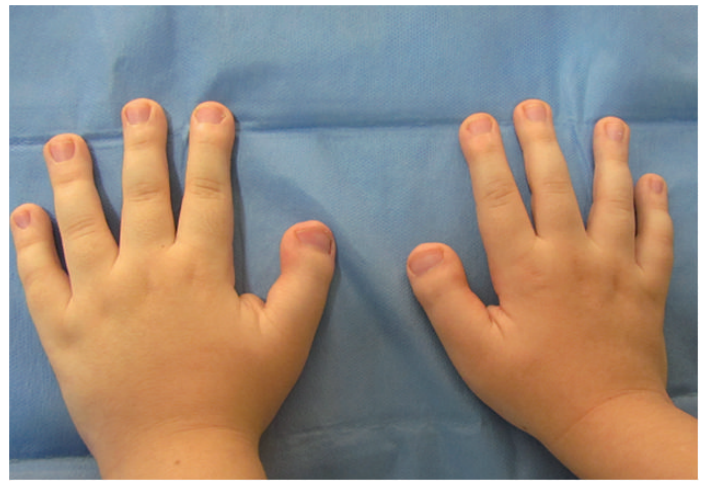

(a)

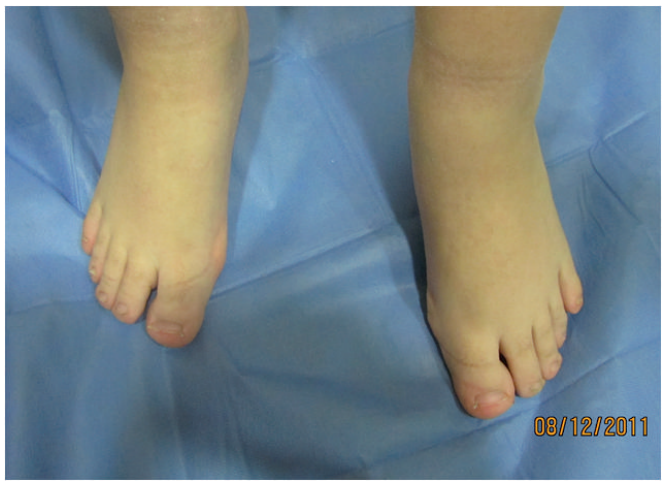

(b)

Figure 1: Hands and feet in RTS. Broad thumbs, broad terminal phalanges were seen.

\section{Case Report}

A 7-year-old girl accompanied by parents reported to the faculty of Dentistry, Department of Pedodontics, Medipol University, Turkey, with a complaint of caries and bleeding of gingiva. She is the second child of a remote consanguineous couple. Her old sister had no signs of dental anomalies. The patient was born at term, weighing $2 \mathrm{~kg} 850 \mathrm{~g}$.

The following clinical extraoral findings were observed: short stature with broad thumbs, mental retardation, down slant of the palpebral fissures, strabismus and simple ear (Figures 1(a), 1(b)). The nose has a beaked appearance, broad fleshy bridge, deviated septum, and short low columella (Figure 2) and she had cardiac abnormalities.

Intra-oral findings were high-arched and cleft palate, a small mouth and malocclusion (Figures 3(a) and 3(b)). Talon cusps were present on maxillary central and lateral incisors (Figure 4). The patient also had carious lesions (Figures 3(a), 3(b), 4, 5(a), 5(b)). Upper and lower molar teeth had deep dentinal carious lesions (no. 16, no. 26, no. 36, no. 46 , no. 75 , no. 85 ). Heavy calculus and plaque deposition could be noted on all teeth. The marginal gingiva was severely swollen and probing resulted in profuse gingival bleeding.

After the complete clinical examination and investigation, the following dental treatment plan was derived.

(1) Oral prophylaxis and topical fluoride application.

(2) Cavity preparation and composite resin restoration (no. 16 , no. 26 , no. 36 , no. 46 , no. 75 , no. 85 ).

(3) Extraction of no. 53, no. 64.

(4) Recall follow-up sessions for every 3 months.

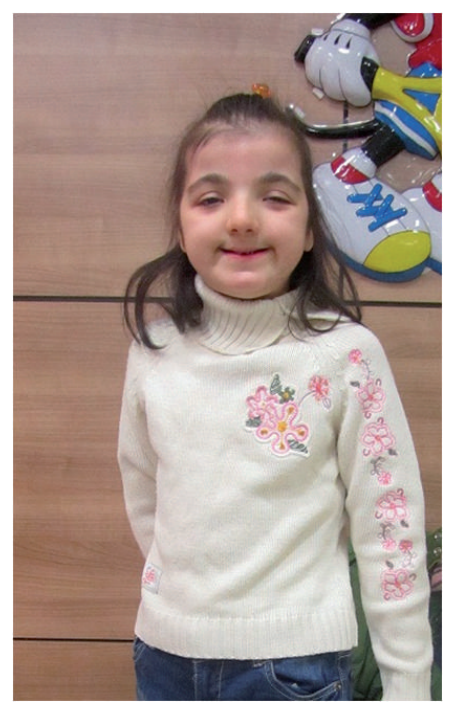

FIGURE 2: Facial characteristics include slanting palpebral fissures and beaked nose with nasal septum.

Due to difficulties in the patient's behavior and her inability to cooperate during dental treatment because of her mental disability, the treatment was performed under general anesthesia.

Detailed oral hygiene instructions were given to her mother who provided routine care for the patient and the patient recalled follow-up sessions every 3 months. However, because of her incoordination about brushing teeth; evidence 


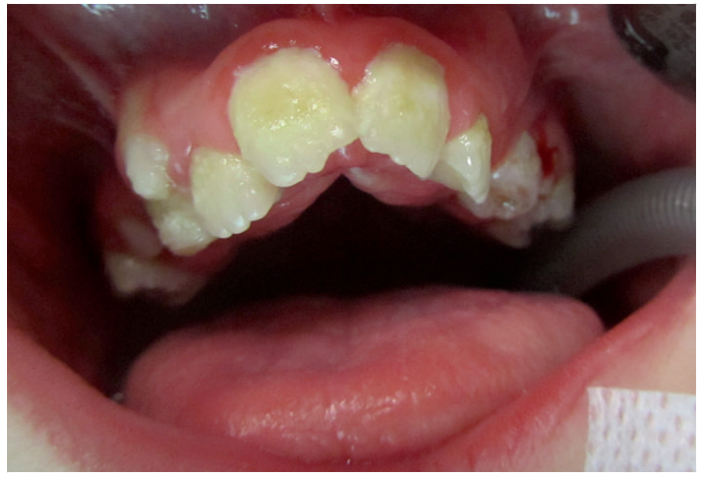

(a)

FIGURE 3: (a) Intraoral findings were a small mouth and malocclusion. (b) In intra-oral findings, high-arched and cleft palate was seen. And upper molar teeth had deep dentinal carious lesions.

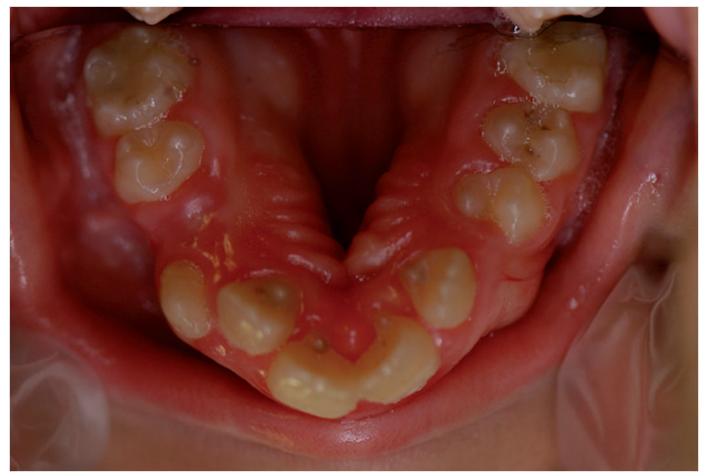

FIGURE 4: Talon cusps were present on maxillary central and lateral incisors.

of unimproved dental hygiene was observed and instead of this no new caries were found. Because of plaque deposition on all teeth, periodontal treatment consisted of scaling, and oral prophylaxis and topical fluoride application were performed under sedation after 6 months later.

\section{Discussion}

The 7-year-old girl described in this paper was diagnosed with RTS associated with a microdeletion at 16p 13.3 chromosome. She had cardiac abnormalities, which are frequently found in $24-38 \%$ cases [ $7,16,19]$.

RTS is a rare multiple congenital anomaly syndrome [20]. Intraoral findings of the patient with RTS were reduced oral opening, a narrow palate, malocclusion, gingivitis, and caries. It was reported that patients with RTS have an increased rate of caries (15\% to $36 \%$ ) because of their poor oral hygiene, which was similar to our patient $[7,12,21]$. Malposition and crowded teeth are present in $62 \%$ of patients $[7,22]$. In this patient, upper and lower molar teeth had deep dentinal carious lesions (no. 16, no. 26, no. 36, no. 46 , no. 75 , no. 85 ). Heavy calculus and plaque deposition could be noted on all teeth. The marginal gingiva was severely swollen and probing resulted in profuse gingival bleeding,

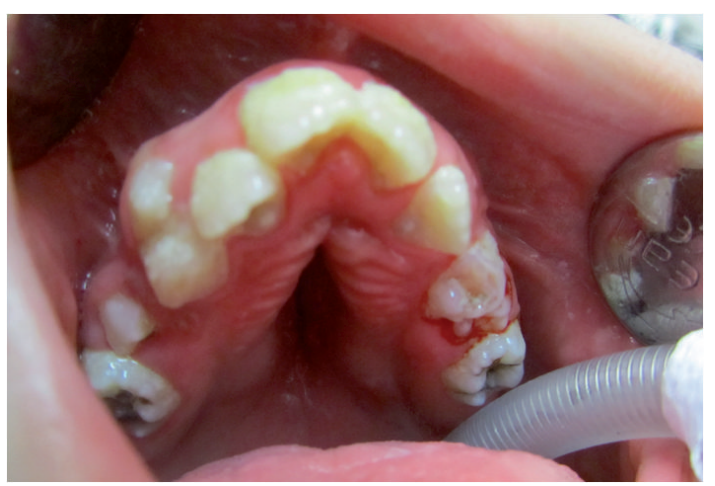

(b)

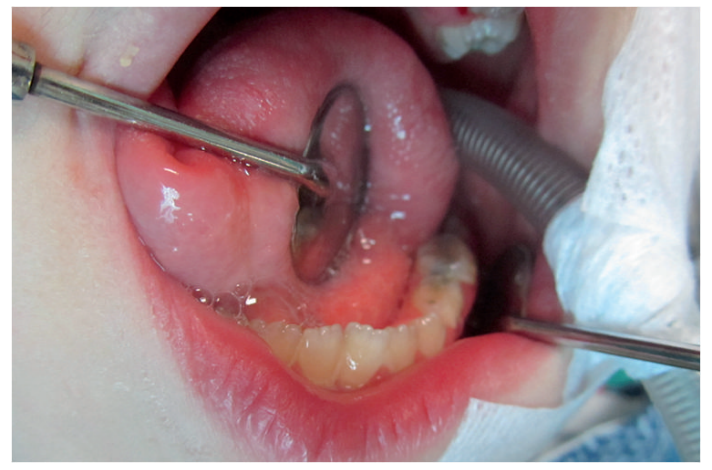

(a)

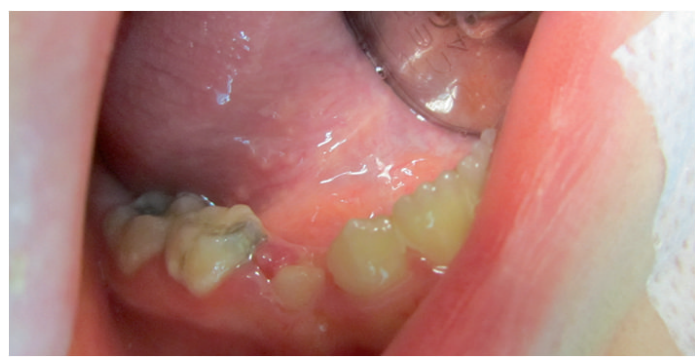

(b)

FIGURE 5: Lower molar teeth had deep dentinal carious lesions.

and malposition was one of, important problems in our patient.

In most patients with this syndrome, it is necessary to carry out the dental treatment under sedation or general anesthesia. It is important to know that these patients might have upper respiratory obstruction during sleeping or sedation because of the anatomical characteristics of their maxillofacial region [16-18]. The treatment of the patient was performed under general anesthesia by anesthesiologist.

Freitas et al. [19] reported the periodontal and immunological status of a 14-year-old female patient with RTS and possible association between syndrome and periodontal disease was reported in the paper. In this paper, heavy calculus 
and plaque deposition could be noted on all teeth. The marginal gingiva was severely swollen and probing resulted in profuse gingival bleeding.

Prospects for future good oral and dental status in this patient are questionable because of her extreme lack of cooperation. For this reason, she needs a longer followup.

\section{References}

[1] J. Michail, J. Matsoukas, and S. Theodorou, "Arched, clubbed thumb in strong abduction-extension \& other concomitant symptoms.", Revue de Chirurgie Orthopédique et Réparatrice de l'Appareil Moteur, vol. 43, no. 2, pp. 142-146, 1957.

[2] J. H. Rubenstein and H. Taybi, "Broad thumbs and toes and facial abnormalities. A possible mental retardation syndrome," American Journal of Diseases of Children, vol. 105, pp. 588-608, 1963.

[3] J. H. Roelfsema and D. J. M. Peters, "Rubinstein-Taybi syndrome: clinical and molecular overview," Expert Reviews in Molecular Medicine, vol. 9, no. 23, pp. 1-16, 2007.

[4] S. H. Kim, B. C. Lim, J. H. Chae, K. J. Kim, and Y. S. Hwang, "A case of Rubinstein-Taybi syndrome with a CREB-binding protein gene mutation," Korean Journal of Pediatrics, vol. 53, no. 6, pp. 718-721, 2010.

[5] A. Bloch-Zupan, J. Stachtou, D. Emmanouil, B. Arveiler, D. Griffiths, and D. Lacombe, "Oro-dental features as useful diagnostic tool in Rubinstein-Taybi syndrome," American Journal of Medical Genetics A, vol. 143, no. 6, pp. 570-573, 2007.

[6] I. Coupry, C. Roudaut, M. Stef et al., "Molecular analysis of the CBP gene in 60 patients with Rubinstein-Taybi syndrome," Journal of Medical Genetics, vol. 39, no. 6, pp. 415-421, 2002.

[7] M. C. Morales-Chávez, "Dental management of a patient with Rubinstein-Taybi syndrome," Special Care in Dentistry, vol. 30, no. 3, pp. 124-126, 2010.

[8] D. G. Gardner and S. S. Girgis, "Talon cusps: a dental anomaly in the Rubinstein-Taybi syndrome," Oral Surgery Oral Medicine and Oral Pathology, vol. 47, no. 6, pp. 519-521, 1979.

[9] R. L. Cirillo, "Pediatric case of the day. RubensteinTaybisyndrome," Radiographics, vol. 17, no. 6, pp. 1604-1605, 1997.

[10] J. E. Ming, E. R. Stiehm, and J. M. Graham Jr., "Immunodefiency as a component of recognizable syndromes," American Journal of Medical Genetics, vol. 66, no. 4, pp. 378-398, 1996.

[11] R. C. Hennekam, "Bibliography on Rubinstein-Taybi syndrome.," American Journal of Medical Genetics. Supplement, vol. 6, pp. 77-83, 1990.

[12] R. W. Miller and J. H. Rubinstein, "Tumors in RubinsteinTaybi syndrome," American Journal of Medical Genetics, vol. 56, no. 1, pp. 112-115, 1995.

[13] C. C. Chuang and H. C. Lin, "Pilomatrixoma of the head and neck," Journal of the Chinese Medical Association, vol. 67, no. 12, pp. 633-636, 2004.

[14] M. J. T. Verstegen, P. Van Den Munckhof, D. Troost, and G. J. Bouma, "Multiple meningiomas in a patient with RubinsteinTaybi syndrome," Journal of Neurosurgery, vol. 102, no. 1, pp. 167-168, 2005.

[15] C. A. Stevens and M. G. Bhakta, "Cardiac abnormalities in the Rubinstein-Taybi syndrome," American Journal of Medical Genetics, vol. 59, no. 3, pp. 346-348, 1995.

[16] S. Wiley, S. Swayne, J. H. Rubinstein, N. E. Lanphear, and C. A. Stevens, "Rubinstein-Taybi syndrome medical guidelines," American Journal of Medical Genetics, vol. 119, no. 2, pp. 101$110,2003$.
[17] L. A. H. Critchley, T. Gin, and J. C. Stuart, "Anaesthesia in an infant with Rubinstein-Taybi syndrome," Anaesthesia, vol. 50, no. 1, pp. 37-38, 1995.

[18] J. E. Allanson, "Rubinstein-Taybi syndrome: the changing face," American Journal of Medical Genetics, no. 6, pp. 38-41, 1990.

[19] N. M. Freitas, A. V. Imbronito, C. S. K. La Scala, R. F. M. Lotufo, and F. E. Pustiglioni, "Periodontal disease in a Rubinstein-Taybi syndrome patient: case report," International Journal of Paediatric Dentistry, vol. 16, no. 4, pp. 292-296, 2006.

[20] A. Stalin, B. Varma, and Jayanthi, "Rubinstein Taybi syndrome," Journal of Indian Society of Pedodontics and Preventive Dentistry, vol. 24, supplement 1, pp. S27-S30, 2006.

[21] S. H. Hsiung, "Rubinstein-Taybi syndrome (broad thumbhallux syndrome)," Dermatology Online Journal, vol. 10, no. 3, article 2, 2004.

[22] E. Davidovich, D. Eimerl, and B. Peretz, "Dental treatment of a child with Rubinstein-Taybi syndrome," Pediatric Dentistry, vol. 27, no. 5, pp. 385-388, 2005. 


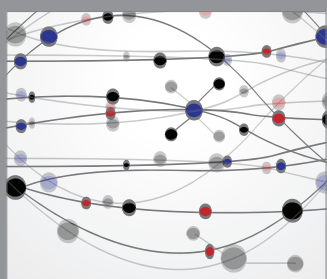

The Scientific World Journal
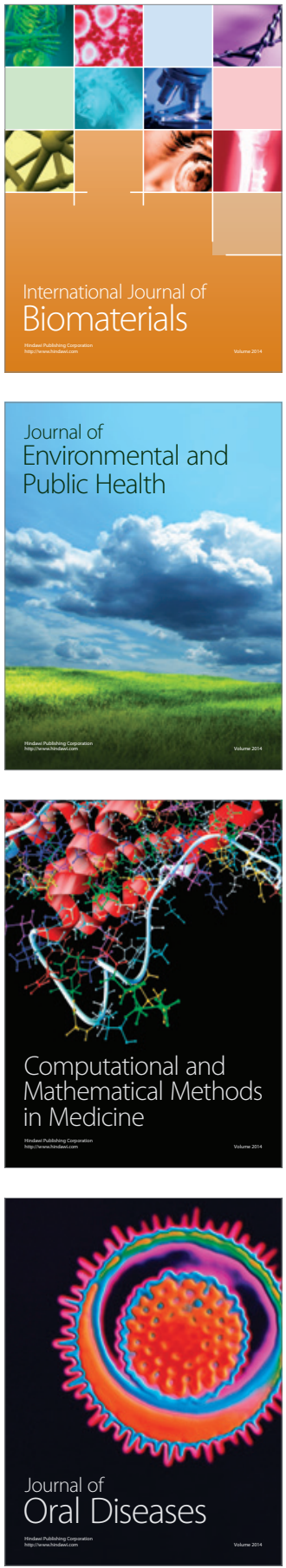
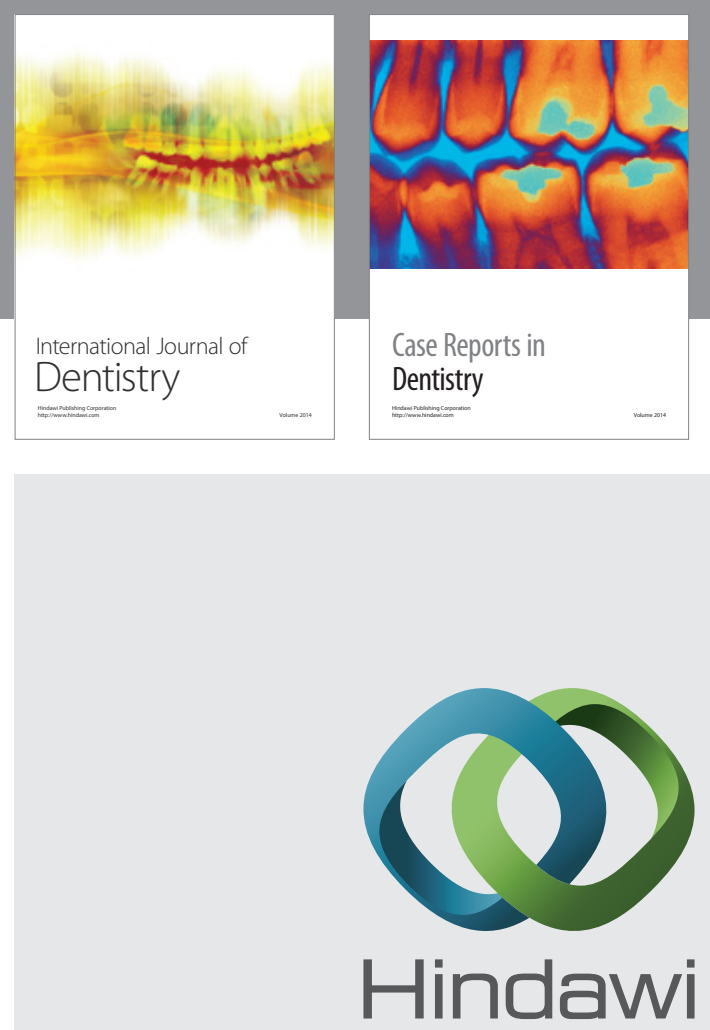

Submit your manuscripts at

http://www.hindawi.com
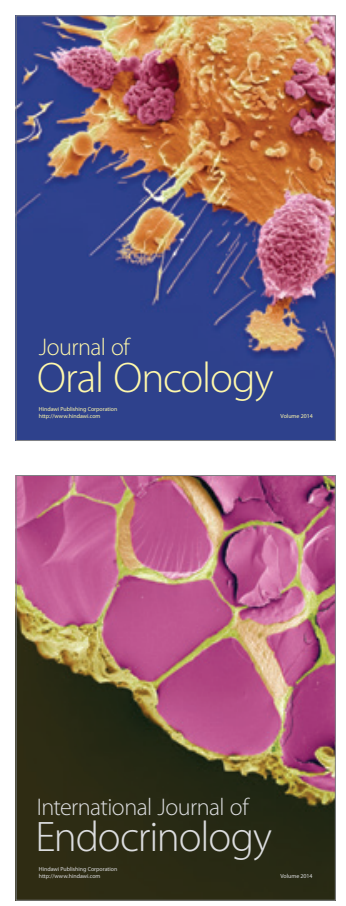
